\title{
Sobrevida e evolução leucêmica de portadores de síndromes mielodisplásicas
}

\section{Survival and leukemic evolution of patients with myelodysplastic syndromes}

\author{
Marcos Antonio Ferreira Júnior¹, Maria Lúcia Ivo², Elenir Rose Cury Jardim Pontes ${ }^{3}$
}

\begin{abstract}
Resumo
As síndromes mielodisplásicas constituem um grupo de doenças de ordem clonal hematopoética evidenciadas por estudos em todo o mundo. A estimativa de sobrevida dos pacientes e de casos que apresentam evolução leucêmica requer investigação na população brasileira, pois não se conta com nenhum dado dessa natureza. Assim, este estudo objetivou caracterizar e estimar a sobrevida e evolução leucêmica de portadores de síndromes mielodisplásicas acompanhados em um serviço de referência. Trata-se de um estudo de coorte retrospectiva realizada de janeiro de 2000 a dezembro de 2010. Para análise descritiva foi utilizado o programa Epi Info 2002, versão 3.5.2, e para os cálculos das probabilidades de sobrevida foi empregado o método de Kaplan-Meier pelo uso do programa Statistic Package for Social Sciences (SPSS), versão 10.0. Os cálculos da probabilidade de associação entre as características analisadas e o gênero foram realizados pelos testes do qui-quadrado de tendência, de Fisher, Mann Whitney, e de Log Rank. O nível de significância considerado foi de 0,05. O trabalho foi aprovado em seus aspectos ético e metodológico pelo Comitê de Ética em Pesquisa do Hospital Universitário Onofre Lopes (HUOL), sob o Protocolo n. 432/10. Dos 29 pacientes selecionados, houve predomínio de idosos, do sexo masculino, com baixa escolaridade. Apresentaram baixa probabilidade acumulada de sobrevida e índices de evolução leucêmica em torno de $27 \%$, sem nenhum resultado satisfatório para o tratamento quimioterápico, bem como nenhuma indicação de transplante de medula óssea como possibilidade de cura. Fazem-se necessárias pesquisas com populações maiores para caracterização em todo território nacional.
\end{abstract}

Palavras-chave: análise de sobrevida; prognóstico; pré-leucemia; anemia refratária; anemia refratária com excesso de blastos; epidemiologia.

\begin{abstract}
Myelodysplastic syndromes constitute a group of clonal hematopoietic diseases shown by studies all around the world. The survival estimation of the patients and the cases presenting leukemic evolution demand investigation in the Brazilian population, as there's no data with regard to this theme. Thus, this study aimed to characterize and estimate the survival and leukemic evolution of patients with myelodysplastic syndromes followed up in a reference service. This is a retrospective cohort study carried out from January 2000 to December 2010. The software Epi Info 2002, version 3.5.2, was used for descriptive analysis, and for the calculations of survival probabilities the Kaplan-Meier method was employed through the software Statistic Package for Social Sciences (SPSS), version 10.0. The calculations of the association probability between the characteristics analyzed and gender were performed using the chi-square for trend, Fisher, Mann Whitney, and Log Rank tests. The significance level was 0.05 . The ethical and methodological aspects of the study were approved by the Research Ethics Committee of HUOL, under the Protocol 432/10. Out of the 29 selected patients, there was a predominance of elderly people, males, with low education. They showed low cumulative probability of survival and leukemic evolution rates around $27 \%$, with no satisfactory outcome from chemotherapy, as well as no indication of bone marrow transplantation as a possible cure. There is a need for researches with larger populations for the characterization all over the national territory.
\end{abstract}

Keywords: survival analysis; prognosis; preleukemia; refractory anemia; anemia, refractory, with excess of blasts; epidemiology.

Trabalho realizado no Hospital Universitário Onofre Lopes - Universidade Federal do Rio Grande do Norte (UFRN) - Natal (RN), Brasil.

'Doutor em Saúde e Desenvolvimento na Região Centro Oeste; Professor Adjunto da UFRN - Natal (RN), Brasil.

2Doutora em Enfermagem Fundamental; Professora Associada da Universidade Federal de Mato Grosso do Sul (UFMS) - Campo Grande (MS), Brasil.

${ }^{3}$ Doutora em Saúde Pública; Professora Adjunta da UFMS - Campo Grande (MS), Brasil.

Endereço para correspondência: Marcos Antonio Ferreira Júnior - Departamento de Enfermagem da Universidade Federal do Rio Grande do Norte -

Avenida Salgado Filho, s/n - Campus Lagoa Nova - CEP: 59072-970 - Natal (RN), Brasil - E-mail: marcos_nurse@hotmail.com

Fonte de financiamento: nenhuma.

Conflito de interesse: nada a declarar. 


\section{INTRODUÇÃO}

As Síndromes Mielodisplásicas (SMD) constituem um grupo diverso de doenças hematopoéticas, de origem clonal, com maior incidência em pacientes idosos ${ }^{1}$. Normalmente são caracterizadas por pancitopenias em sangue periférico e uma medula óssea normo ou hipercelular, com alterações displásicas de tamanho, forma e organização celular, podendo acumular células imaturas denominadas blastos leucêmicos. Foram denominadas apenas como anemia refratária ao longo da prática hematológica, mas, em razão do curso desses quadros, houve a necessidade de melhor detalhamento de cada caso desse grupo tão heterogêneo de displasias hematopoéticas ${ }^{2-4}$.

Os quadros clínicos são os mais diversos, podendo variar de situações de anemias leves e crônicas até aqueles com uma acentuada redução do número das células sanguíneas e aparecimento de blastos leucêmicos nas amostras medulares, constituindo os quadros mais agudos e graves. A doença pode progredir também de tal forma que os blastos leucêmicos tomam conta da medula óssea e evolui para um quadro de Leucemia Mieloide Aguda (LMA) ${ }^{5}$. Por tempos, a doença foi conhecida como pré-leucêmica em razão dos casos que apresentaram evolução, porém, com as classificações internacionais para as SMD, realizadas inicialmente pelo Grupo Franco-AmericanoBritânico (FAB) e, após, pela Organização Mundial de Saúde (OMS), têm-se buscado desmistificar o caráter oncológico da doença, de forma a melhorar cada vez mais o diagnóstico e estratificar condutas terapêuticas para cada subtipo descrito ${ }^{6-10}$.

Sabe-se que a incidência das SMD é crescente, de acordo com a idade, variando de 4,1 a 12,6 novos casos/100.000 habitantes/ano, com aumento para cerca de 90 novos casos/100.000 habitantes/ano na população com idade superior a 80 anos $^{11,12}$. Porém, a sobrevida desses pacientes não é bem definida, principalmente em relação a dados nacionais ${ }^{11-13}$.

Nesse sentido, devido à inexistência de estudos sobre sobrevida e evolução leucêmica dos pacientes portadores de SMD no Brasil, objetivou-se caracterizar e estimar a sobrevida e evolução leucêmica de pacientes acompanhados em um Serviço de Referência.

\section{MATERIAL E MÉTODOS}

\section{População de estudo}

Estudou-se uma coorte retrospectiva de base hospitalar e ambulatorial, incluindo pacientes com diagnóstico de Síndromes Mielodisplásicas, atendidos pelo Serviço de Hematologia de um hospital de referência de alta complexidade no Estado do Rio Grande do Norte, no período de janeiro de 2000 a dezembro de 2010.

\section{Coleta de dados}

Para o levantamento dos sujeitos da pesquisa não foi possível utilizar nenhum banco de dados informatizado para triagem, uma vez que o serviço analisado não dispunha de informatização cadastral que possibilitasse filtrar especificamente a amostra em estudo. Diante disso, foram analisados todos os laudos das biópsias de medula óssea, realizadas no período, junto ao Serviço de Patologia, para a partir desses laudos caracterizar a população a ser estudada. A análise dos exames de biópsia de medula óssea se deu inicialmente pela verificação dos livros de registro de todas as biópsias realizadas, com busca por aquelas solicitadas pelo Serviço de Hematologia e somente a partir daí constituir a amostra final para início da análise.

O Serviço de Patologia realizou um total de 51.277 exames de biópsias durante o período. A menor frequência de solicitação de exames foi originada pelo Serviço de Hematologia. Foram solicitados 184 exames de biópsias de medula óssea, que corresponderam a $0,35 \%$ do total. Após a análise dos laudos, foram selecionados aqueles sugestivos da doença em estudo.

Do total, 115 apresentaram laudos sugestivos de displasias medulares, alguns mais conclusivos para as SMD e outros apenas com detecção de hiperproliferação ou hipoproliferação celular, aplasias, leucoses, infiltrações medulares por células redondas ou tecidos adiposos, bem como sugestivos de evolução leucêmica. Os laudos confirmatórios de leucemia também foram incluídos para análise do prontuário, a fim de verificar casos iniciais de SMD com evolução leucêmica. Após essa seleção inicial, foram analisados todos os prontuários junto ao Serviço de Arquivamento Médico e Estatística para avaliação do diagnóstico confirmado de SMD e coleta de dados por meio de formulário próprio.

Foram incluídos na amostra pacientes com diagnóstico médico de SMD, independente do sistema de classificação adotado, de ambos os sexos, em atendimento ou já atendidos, e de todas as idades. Foram excluídos aqueles com diagnóstico não confirmado, apesar do laudo sugestivo da biópsia, bem como os que não contiveram informações nos prontuários capazes de fornecer dados necessários ao estudo. A amostra final foi de 29 pacientes. O período de coleta de dados se deu entre outubro de 2010 e fevereiro de 2011.

\section{Variáveis do estudo}

Foram analisadas as datas dos diagnósticos, o tempo despendido para procura pelo serviço referenciado após início dos sintomas, o tempo de acompanhamento depois de admitido no serviço e o tempo de encerramento para os casos de óbito, bem como sua causa. Para fins de cálculo de sobrevida foi dado como tempo zero o momento do diagnóstico. 
O encerramento foi caracterizado pelo óbito, evolução leucêmica ou abandono. A evolução leucêmica foi caracterizada por tempo de evolução, gênero e idade.

\section{Análise dos dados}

A entrada dos dados foi realizada por meio do programa Microsoft Excel - Windows 95 para procedimentos de análises descritivas e inferenciais. Para análise descritiva dos dados foi utilizado o programa Epi Info 2002, versão 3.5.2. Para descrição da amostra, foram utilizadas tabelas contendo frequências absolutas e relativas por gênero e total, médias e desvios-padrão. Para cálculo da probabilidade de associação entre as características analisadas e o gênero foram utilizados o teste do $\chi^{2}$ de tendência, o teste de Fisher e o teste de MannWhitney, de acordo com cada caso ${ }^{14}$. Os cálculos de sobrevida foram determinados pelo Método de Kaplan-Meier, considerando como marco inicial a data do diagnóstico e, final, o último evento: óbito, evolução leucêmica ou abandono, num período de onze anos. Para comparação das sobrevidas foi utilizado o método estatístico de $\log \operatorname{Rank}^{15}$. Os cálculos de sobrevida foram realizados com uso do software Statistic Package for Social Sciences (SPSS), versão 10.0. O nível de significância considerado foi de 0,05.

\section{Apreciação ética}

O protocolo de pesquisa do estudo foi submetido ao Comitê de Ética em Pesquisa do Hospital Universitário Onofre Lopes, da Universidade Federal do Rio Grande do Norte (CEP/HUOL/UFRN), de acordo com as exigências da Resolução nº 196/1996 do Conselho Nacional de Saúde, e aprovado em seus aspectos éticos e metodológicos, conforme o Certificado do Protocolo CEP/HUOL de número 432/10.

\section{RESULTADOS}

Dos 29 pacientes, $17(58,6 \%)$ eram homens e $12(41,3 \%)$ mulheres. A idade dos pacientes variou de 22 a 86 anos, com média de 57,34 anos. Para o sexo feminino a idade variou de 23 a 72 anos (média de 53,25 anos); para o sexo masculino a variação foi de 22 a 86 anos (média de 60,23 anos). No total, $65,6 \%$ apresentaram idade igual ou superior a 50 anos e $51,7 \%$ acima de 60 anos.

Cerca de $69 \%$ eram casados ou viviam em união estável. A maior parte $(44,8 \%)$ possuía ensino fundamental incompleto ou analfabetismo, com parte significativa $(24,1 \%)$ de analfabetos. Houve um predomínio da cor de pele branca $(82,7 \%)$, possuidores de casa própria $(72,4 \%)$ e moradores na zona urbana $(89,7 \%)$. A renda familiar de até dois salários mínimos correspondeu a 72,4\%. Em relação à ocupação,
$79,3 \%$ eram de trabalhadores do comércio ou prestadores de serviços. Cerca de $51 \%$ residiam na capital, com o restante no interior do Estado do Rio Grande do Norte.

Considerando-se as características clínicas, somente um caso $(3,4 \%)$ de SMD foi classificado como SMD secundária, frente a 28 casos $(96,6 \%)$ de SMD primárias. A evolução temporal dos pacientes buscou evidenciar os tempos de procura pelo serviço que os pacientes despenderam desde o início dos sintomas, bem como os tempos de acompanhamento. Em relação a esses períodos, não foi observada diferença estatisticamente significativa entre os gêneros.

O tempo de procura variou desde dois meses até quatro anos após o início dos sintomas. Cerca de 31\% dos casos procuraram o serviço com um intervalo superior a 12 até 24 meses, seguidos por $27,6 \%$ com até dois meses de espera. A média geral do tempo de procura foi de 13,5 meses. Já o tempo de acompanhamento dos pacientes teve maior frequência entre o intervalo de até dois meses $(31,1 \%)$, seguido do intervalo de 48 até 120 meses (27,6\%), com média de 47,4 meses.

Em relação ao tempo de encerramento por óbito $(n=17)$, foi mais frequente o intervalo até seis meses (58,8\%), com metade deste até um mês. A média de tempo para encerramento por morte foi de 19,1 meses. Apresentou diferença estatisticamente significativa na comparação por gênero $(\mathrm{p}=0,045)$, com deteç̧ão de óbitos mais precoces para o sexo feminino (2,6 meses) em relação ao masculino (26,0 meses). Ainda em relação aos encerramentos por óbito, as principais causas descritas nos atestados de óbito foram Parada Cardiorrespiratória (82,35\%) e Falência de Múltiplos Órgãos (41,2\%), sem diferenças entre gêneros (Tabela 1).

A probabilidade acumulada de sobrevida foi calculada a partir do total de pacientes em estudo $(n=29)$, sendo destes: 17 óbitos (58,6\%), 11 em tratamento (37,9\%) e 01 abandono $(3,4 \%)$. O tempo zero (inicial) foi considerado o momento do diagnóstico. Portanto, com um mês, a probabilidade acumulada de sobrevida foi de $72 \%$; com dois meses, $62 \%$; com dez meses, 58\%; com um ano, 55\%; com dois anos $51 \%$ e com dez anos 24\%. Entre homens e mulheres não houve diferença estatisticamente significativa $(\log \operatorname{Rank}=0,68)$ (Tabela 2). Observou-se uma curva de sobrevida maior nas mulheres em relação aos homens, porém, o método estatístico de Log Rank não alcançou valor crítico que permitisse estabelecer diferença estatisticamente significativa entre os grupos (Figuras 1 e 2).

Do total de pacientes, oito (27,5\%) apresentaram evolução leucêmica a partir da SMD, sendo, destes, cinco homens e três mulheres. Não houve diferença estatística dos quadros evolutivos em relação à idade ou ao gênero, apenas maiores concentrações de casos entre as faixas etárias de 30 a 40 anos ( 2 casos) e de 70 a 80 anos ( 2 casos) (Tabela 3 ). 
Das evoluções leucêmicas, todas apresentaram a LMA como progressão. O tempo entre o diagnóstico confirmado de SMD e a evolução leucêmica variou de 4 a 48 meses, com um tempo médio de evolução de 15,2 meses. O tratamento quimioterápico foi indicado em $62,5 \%$ dos casos, sendo todos com resultados insatisfatórios, culminados em óbito. Não houve diferença estatística significativa para nenhumas dessas características. Nenhum dos pacientes com evolução leucêmica teve indicação de realização de transplante de medula óssea.

Tabela 1. Distribuição de óbitos de pacientes portadores de síndromes mielodisplásicas segundo o tempo de encerramento e causas de morte, Natal/RN - 2011

\begin{tabular}{|c|c|c|c|c|c|c|c|}
\hline \multirow[t]{2}{*}{ Variáveis } & \multicolumn{2}{|c|}{$\begin{array}{c}\text { Masculino } \\
(\mathrm{n}=12)\end{array}$} & \multicolumn{2}{|c|}{$\begin{array}{l}\text { Feminino } \\
\quad(n=5)\end{array}$} & \multicolumn{2}{|c|}{$\begin{array}{c}\text { Total } \\
(n=17)\end{array}$} & \multirow[t]{2}{*}{ Valor $\mathrm{p}$} \\
\hline & $\mathbf{n}$ & $\%$ & n & $\%$ & $\mathrm{n}$ & $\%$ & \\
\hline \multicolumn{8}{|l|}{ Tempo de Encerramento ${ }^{a}$} \\
\hline Até um mês & 2 & 16,7 & 3 & 60,0 & 5 & 29,4 & \\
\hline$>1$ até 6 meses & 3 & 25,0 & 2 & 40,0 & 5 & 29,4 & - \\
\hline$>6$ até 12 meses & 2 & 16,7 & - & - & 2 & 11,8 & \\
\hline$>12$ até 24 meses & 2 & 16,7 & - & - & 2 & 11,8 & \\
\hline$>24$ até 120 meses & 3 & 25,0 & - & - & 3 & 17,6 & \\
\hline Média \pm desvio padrão & \multicolumn{2}{|c|}{$26,0 \pm 36,1$} & \multicolumn{2}{|c|}{$2,6 \pm 2,2$} & \multicolumn{2}{|c|}{$19,1 \pm 31,9$} & $0,045^{\mathrm{c}}$ \\
\hline \multicolumn{8}{|l|}{ Causas de morte $\mathrm{e}^{\mathrm{b}}$} \\
\hline Falência de múltiplos órgãos & 6 & 50,0 & 1 & 20,0 & 7 & 41,2 & $0,338^{\mathrm{d}}$ \\
\hline Parada cardiorrespiratória & 9 & 75,0 & 5 & 100,0 & 14 & 82,35 & $0,515^{\mathrm{d})}$ \\
\hline Acidente Vascular Encefálico & 1 & 8,4 & - & - & 1 & 5,9 & $1,000^{\mathrm{d}}$ \\
\hline Sepse & - & - & 2 & 40,0 & 2 & 11,8 & $0,074^{\mathrm{d}}$ \\
\hline Leucemia & - & - & 1 & 20,0 & 1 & 5,9 & $0,294^{\mathrm{d}}$ \\
\hline
\end{tabular}

${ }^{a}$ Encerramentos com óbito durante o período em estudo; 'be acordo com atestado médico de óbito constante nos prontuários. Cada atestado poderia conter uma ou mais causas de morte; cteste de Mann-Whitney; dteste de Fisher.

Tabela 2. Probabilidade Acumulada de Sobrevida dos pacientes portadores de Síndromes Mielodisplásicas. Natal/RN - 2011 ( $\mathrm{n}=29$ )

\begin{tabular}{lccc} 
Tempo de Acompanhamento & $\begin{array}{c}\text { Probabilidade Acumulada de } \\
\text { (meses) }\end{array}$ & $\begin{array}{c}\text { Masculino } \\
\mathbf{n}=\mathbf{1 7}\end{array}$ & $\begin{array}{c}\text { Feminino } \\
\mathbf{n}=\mathbf{1 2}\end{array}$ \\
\cline { 3 - 4 } 1 & 0,724 & 0,765 & 0,667 \\
2 & 0,621 & 0,647 & 0,583 \\
11 & 0,621 & 0,588 & - \\
12 & 0,552 & 0,529 & - \\
24 & 0,517 & 0,471 & - \\
27 & 0,480 & 0,412 & - \\
112 & 0,360 & - & 0,292 \\
120 & 0,240 & 0,206 & - \\
\hline
\end{tabular}

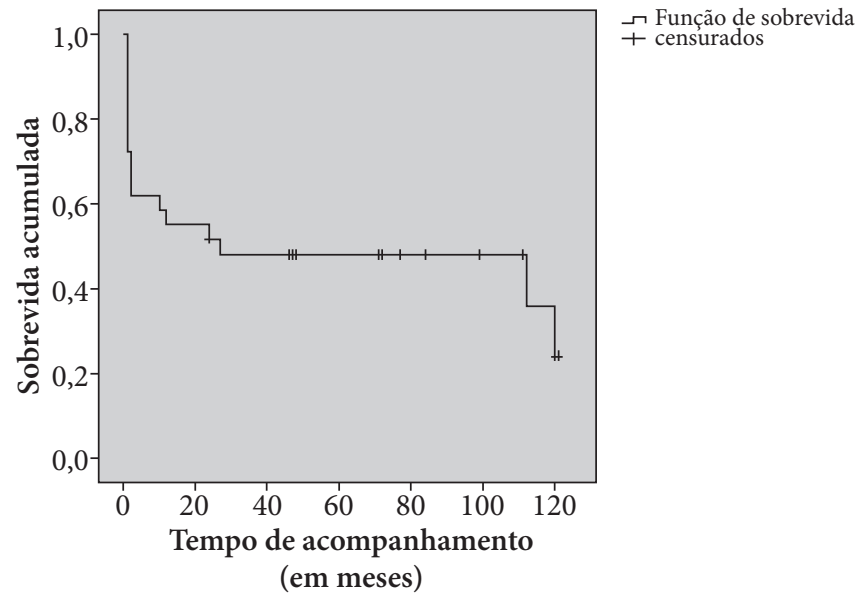

Figura 1. Curva de sobrevivência da coorte estudada entre 2000 e $2010(n=29)$

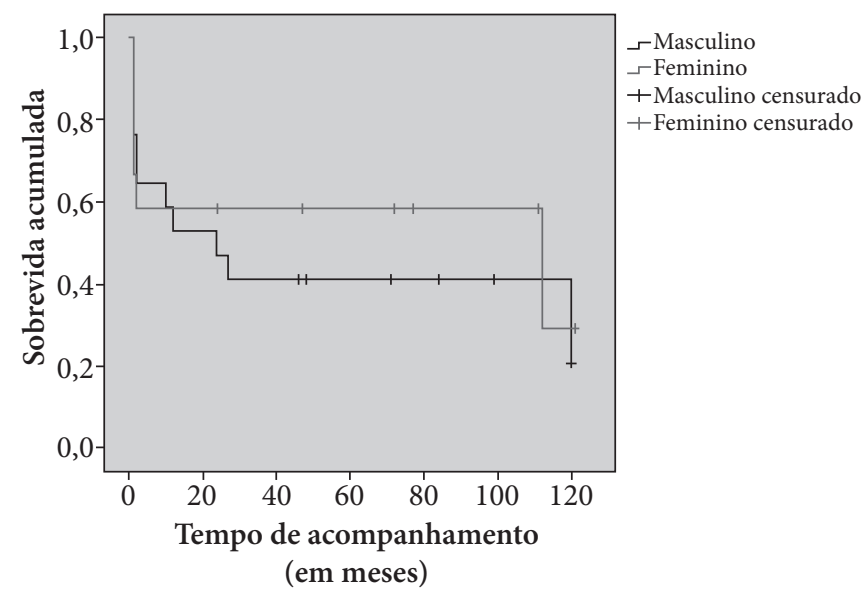

Figura 2. Curva de sobrevivência por gênero da coorte estudada entre 2000 e 2010 ( $n=29)$ 
Tabela 3. Distribuição de pacientes portadores de Síndromes Mielodisplásicas segundo evolução leucêmica, gênero e faixa etária, Natal/RN - 2011 ( $n=29)$

\begin{tabular}{lccccc} 
& \multicolumn{5}{c}{ Evolução leucêmica } \\
\cline { 2 - 4 } Variáveis & \multicolumn{2}{c}{$\operatorname{Sim}(\mathbf{n}=\mathbf{8})$} & \multicolumn{2}{c}{ Não $(\mathbf{n}=\mathbf{2 1})$} & \multirow{2}{*}{ Valor p } \\
\cline { 2 - 4 } & $\mathbf{n}$ & $\%$ & $\mathbf{n}$ & $\%$ & \\
Gênero & & & & & \\
Masculino & 5 & 29,4 & 12 & 70,6 & $1,000^{\mathrm{a}}$ \\
Feminino & 3 & 25,0 & 9 & 75,0 & \\
Faixa etária & & & & & \\
$\quad<30$ anos & 1 & 33,3 & 2 & 66,7 & \\
$30<40$ anos & 2 & 50,0 & 2 & 50,0 & \\
$40<50$ anos & 1 & 33,3 & 2 & 66,7 & \\
$50<60$ anos & 1 & 25,0 & 3 & 75,0 & $0,205^{\mathrm{b}}$ \\
$60<70$ anos & 1 & 25,0 & 3 & 75,0 & \\
$70<80$ anos & 2 & 25,0 & 6 & 75,0 & \\
$\geq 80$ anos & - & - & 3 & 100,0 & \\
\hline
\end{tabular}

aTeste de Fisher; bteste do $\chi^{2}$ de tendência.

\section{DISCUSSÃO}

Estudos de sobrevida e evolução leucêmica para as SMD, com amostras de bases populacionais grandes, são recentes e realizados geralmente em países europeus ou norte-americanos. Destacam-se um primeiro estudo francês ${ }^{16}$, outro sobre incidência e resultados no sudeste da Inglaterra ${ }^{17}$, e um norte-americano sobre incidência e sobrevida nos Estados Unidos da América - EUA ${ }^{18}$. Também merece destaque um estudo alemão anterior ${ }^{19}$, porém, com menor amostra. No Brasil, nenhum estudo com abordagem epidemiológica para caracterização do paciente portador de SMD e estimativa de sua sobrevida ou evolução leucêmica foi publicado ainda.

Esse presente estudo caracterizou inicialmente a amostra e encontrou uma idade média de 57,3 anos para os casos. Esta foi menor que a encontrada nos EUA (76 anos), Alemanha (72 anos) e Inglaterra (69 anos), porém, acima da Coreia, Japão e China (57,2; 57,1 e 53,2 anos, respectivamente $)^{16-19}$. A população estudada ficou em quarto lugar de acordo com a média de idade decrescente. A relação de homem/mulher de 1,4 converge com a maior parte dos estudos que estabelece um predomínio do sexo masculino, bem como da idade acima de 60 anos $^{18}$.

O tempo médio de espera desde o aparecimento dos sintomas até a procura pelo serviço foi de 13,5 meses, próximo ao encontrado nos EUA, que foi de 11,2 meses $^{18}$. Já o tempo de acompanhamento foi de aproximadamente quatro anos (47,4 meses), próximo dos 50 meses da França ${ }^{16}$ e dos 42 meses nos EUA ${ }^{18}$; e baixo em relação aos 60 meses da Inglaterra ${ }^{17}$.

A diferença estatisticamente significativa encontrada em relação ao gênero que atribuiu às mulheres o óbito mais precoce, não se confirmou por nenhum dos estudos comparados, possivelmente pelo tamanho da amostra. As causas de morte não foram relatadas em nenhum dos estudos epidemiológicos com grandes amostragens referenciados.

A probabilidade acumulada de sobrevida detectada nesse estudo (cerca de $48 \%$ em 5 anos) foi próxima da encontrada por Maynadié $(45 \%)^{16}$, maior que as de Phekoo $(32 \%)^{17}$ e de $\mathrm{Ma}(42 \%)^{18}$, no mesmo período. Porém, os estudos comparados puderam fazer curvas de sobrevida para cada subtipo de SMD, pois os sistemas de informação utilizados forneceram os diagnósticos estratificados, o que não foi possível neste estudo. No estudo inglês e norte-americano foi detectada diferença estatística entre os gêneros, com uma sobrevida acumulada menor para os homens ${ }^{18,19}$; já neste estudo não houve essa diferença apesar do observado na Figura $2(\log \operatorname{Rank}=0,612)$. $\mathrm{O}$ estudo francês confirma o pior prognóstico para aqueles casos classificados como Anemia Refratária com Excesso de Blastos em transformação (AREB-t) e Anemia Refratária com Sideroblastos em Anel (ARSA) ${ }^{16}$.

A evolução leucêmica encontrada ( $27,5 \%$ dos casos) foi menor que a francesa de $31 \%^{16}$. O estudo francês fez o quadro evolutivo por subtipo da classificação das SMD, que não foi possível neste estudo por não fornecer o diagnóstico classificado, e encontrou $44 \%$ de transformação para Anemia Refratária com Excesso de Blastos em transformação (AREB-t), 39\% para Anemia Refratária com Excesso de Blastos (AREB), 30\% para Leucemia Mielomonocítica Crônica (LMMC), 17\% para Anemia Refratária (AR) e 7\% para Anemia Refratária com Sideroblastos em Anel (ARSA), segundo a classificação FAB ${ }^{16}$.

As evoluções leucêmicas foram em sua totalidade para LMA, da mesma forma que no estudo francês, quando todos os casos evoluíram exclusivamente para o mesmo tipo, e os demais estudos não atribuíram notas para essas evoluções. O tempo médio de evolução das SMD para quadros leucêmicos detectados foi de 15,2 meses, próximo ao tempo francês de 18,4 meses $^{16}$.

O tratamento quimioterápico foi indicado em $62,5 \%$ dos casos com evolução leucêmica, porém, os estudos disponíveis apontam que os casos de SMD com início progressivo para leucemia apresentam melhor resposta ao tratamento; entretanto, os resultados dependem da evolução da doença. Cerca de 77 a $86 \%$ de sucesso é atingido para aqueles com menos de seis meses da doença e de 25 a $28 \%$ para aqueles com mais de meio ano. As taxas de mortalidade dos pacientes com SMD submetidos à quimioterapia são de 0 a $10 \%{ }^{20}$. Todos os casos do estudo tratados com quimioterapia vieram a óbito, provavelmente pela introdução da terapia tardiamente.

O transplante de medula óssea não foi indicado em nenhum dos casos, porém, parece ser um recurso com possibilidade de cura a cerca de $40 \%$ dos casos por meio do transplante alogênico. Os melhores resultados são referenciados 
aos portadores de SMD classificadas como subtipo Anemia Refratária, com uma sobrevida livre de recidivas de até $65 \%$ em $5 \operatorname{anos}^{21}$.

As dificuldades para realizar o diagnóstico da maior parte dos centros hematológicos brasileiros, que diferem dos grandes centros de pesquisa, junto à subnotificação das SMD e a falta de informações adequadas nos prontuários para realização de inquéritos investigativos, bem como sistemas de informação inadequados nos setores de hematologia, constituem as limitações deste estudo em relação ao número de sujeitos necessários para pesquisas com maiores populações.
Entretanto, é possível afirmar que os pacientes são na maioria idosos, predominantemente do sexo masculino, com probabilidades de sobrevida baixas, com índices de evolução leucêmica compatíveis com estudos de outros países. Este estudo consiste num ponto de partida para as taxas de incidência e sobrevida, bem como evolução leucêmica dos pacientes portadores de SMD no Brasil. Portanto, é necessária a realização de mais estudos, com populações maiores, de caráter multicêntrico, de forma a estimar a sobrevida dos portadores de SMD e os casos evolutivos por regiões no Brasil, e apontar áreas prioritárias para investigações.

\section{REFERÊNCIAS}

1. Chauffaille MLLF. Alterações cromossômicas em síndrome mielodisplásica. Rev Bras Hematol Hemoter. 2006;28(3):182-7.

2. Geary CG, Macheta AT. Myelodysplasia and preleukaemia. In: Leukaemia and related disorders. Whittaker JA and Holmes JA. 3th edition. Blackwell Science Ltd, Oxford, 1998, p. 195-228.

3. Lowenthal RM, Marsden KA. Myelodysplastic syndromes. Int J Hematol 1997;65(3):318-38.

4. Steensma DP, Bennett JM. The Myelodysplastic syndromes: diagnosis and treatment. Mayo Clin Proc. 2006;81(1):104-30.

5. Magalhães SMM. Síndromes mielodisplásicas - diagnóstico de exclusão. Rev Bras Hematol Hemoter. 2006;28(3):175-7.

6. The Myelodysplastic Syndromes Foundation's Awareness program for 2005-2006. Understanding MDS - a Primer for Practicing Clinicians, segment 1 and 2 .

7. Apa AG, Gutz CNRM. Fatores prognósticos nas síndromes mielodisplásicas. Rev Bras Hematol Hemoter. 2006;28(3):198-200.

8. Bennet JM, Catovsky D, Daniel MT, et al. Proposals for the classification of the myelodysplastic syndromes. Br J Haematol. 1982;51:189-99.

9. Vardiman JW, Harris NL, Brunning RD. The Word Health Organization (WHO) classification of myeloid neoplasms. Blood. 2002;100:2292-302.

10. Moraes ACR, Licínio MA, Pagnussat L, Del Moral JAG, Santos-Silva MC. Síndromes mielodisplásicas: aspectos moleculares, laboratoriais e a classificação OMS 2008. Rev Bras Hematol Hemoter. 2009;31(6):463-70.

11. Magalhães SMM, Lorand-Metze I. Síndromes mielodisplásicas protocolo de exclusão. Rev Bras Hematol Hemoter. 2004;26(4):263-7.

12. Aul C, Gattermann N, Schneider W. Epidemiological and etiological aspects of myelodysplastic syndromes. Leuk Lymphoma. 1995;16:247-62.
13. Chang G, Meadows ME, Jones JA, Antin JH, Orav EJ. Substance Use and Survival after Treatment for Chronic Myelogenous Leukemia (CML) or Myelodysplastic Syndrome (MDS). Am J Drug Alcohol Abuse. 2010;36(1):1-6.

14. Callegari-Jacques SM. Bioestatística: princípios e aplicações. Porto Alegre: Artmed; 2003.

15. Dawson B, Trapp RG. Análise dos temas de pesquisa sobre sobrevivência. In: Dawson B, Trapp RG. Bioestatística básica e clínica. Rio de Janeiro: McGrawHill; 2003. p.187-205.

16. Maynadie M, Verret C, Moskovtchenko P, Mugneret F, Petrella T, Caillot $\mathrm{D}$, et al. Epidemiological characteristics of myelodysplastic syndrome in a well-defined French population. Br J Cancer. 1996;74(8):288-90.

17. Phekoo KJ, Richards MA, Moller H, Schey AS. The incidence and outcome of myeloid malignancies in 2.112 adult patients in south EastEngland. Haematologica. 2006;91(10):1400-4.

18. Ma X, Does M, Raza A, Mayne ST. Myelodysplastic syndromes: incidence and survival in the United States. Cancer. 2007;109(8):1536-42.

19. Aul C, Gattermann N, Schneider W. Age-related incidence and other epidemiological aspects of myelodysplastic syndromes. Br J Haematol. 1992;82:358-67.

20. Cortes J, Kantarjian H, Albitar M, Thomas D, Faderl S, Kokker C, et al A randomized trial of liposomal daunorubicim and cytarabine versus liposomal daunorubicin and topotecan with or without thalidomide as initial therapy for patients with poor prognosis acute myelogenous leukemia or myelodysplastic syndromes. Cancer. 2003;97(1):234-41.

21. Tabak DG, Pereira SCM, Nogueira MC. Transplante de célula-tronco para síndrome mielodisplásica. Rev Bras Hematol Hemoter. 2010;32(Suppl 1):66-70.

Recebido em: 23/10/2012 Aprovado em: 15/05/2013 